\title{
A case of complete heart block with a therapeutic dilemma
}

\author{
Authors: Tomislav Cigić ${ }^{1}$, Duje Čulina1, Vjekoslav Radeljić ${ }^{2}$ \\ 1 School of Medicine, University of Zagreb, Croatia \\ ${ }^{2}$ University Hospital Centre Sestre milosrdnice, Clinic for Cardiovascular diseases, Zagreb, Croatia \\ DOI: https://doi.org/10.26800/LV-142-supp5-4
}

\section{Background:}

Third-degree atrioventricular (AV) block, also referred to as complete heart block (CHB), is caused by a cardiac conduction system disease or damage resulting in no conduction through the atrioventricular node (AVN), leading to complete dissociation of the atrial and ventricular beats. We present a case of a patient with acquired $\mathrm{CHB}$ who has been treated without a permanent pacemaker.

\section{Case presentation:}

A 28-year-old female patient felt palpitations without any previous pre/syncopae. The patient was not taking any medications and had no positive family history for conduction disturbance nor arrhythmias. Physical examination revealed no pathology with a resting heart rate of 56/minute. However, 12-lead ECG revealed CHB with the narrow QRS complex. Laboratory findings ruled out systematic auto/inflammatory and/or connective tissue diseases, as well as infectious diseases and malignancies. Stress-ECG testing showed symptomatic CHB with a maximum heart rate of 110 /minute. Echocardiography revealed the normal systolic and diastolic function of both ventricles without valvular or structural abnormalities. Cardiac magnetic resonance ruled out myocarditis and structural heart disease. During the diagnostic workout, symptoms started to disappear and 12-lead ECG revealed AVN conduction recovery with $P Q$ interval prolongation (300-340 ms). Furthermore, second stressECG testing was done showing normal $A V$ node conduction, $P Q$ interval reduction, narrow $Q R S$, and maximal heart rate of $180 /$ minute. Thus, the patient was discharged without permanent pacemaker implantation although this was against international guidelines recommendation, due to the AV node conduction recovery and patient being asymptomatic.

\section{Conclusion:}

In conclusion, implanting a permanent pacemaker is a class I indication for all symptomatic patients with $\mathrm{CHB}$. However, in this case, we are pointing that watchful waiting while doing a diagnostic workout of acquired $\mathrm{CHB}$, is reasonable since several etiologies can be transient or easily curable. The patient, who had no hemodynamic imbalances or syncopae, has no indication for emergency pacemaker implantation.

Keywords: complete heart block (CHB), conservative treatment, palpitations, permanent pacemaker 